Philosophie ANTIQUE

\section{Philosophie antique}

Problèmes, Renaissances, Usages

$11 \mid 2011$

Influences, filiations, réceptions (XVIIe-XXe siècles)

\title{
Jan Łukasiewicz, Sur la Logique des stoïciens
}

\author{
Jan Wolenski
}

Traducteur : Anna Zielinska

\section{CpenEdition}

\section{Journals}

Édition électronique

URL : http://journals.openedition.org/philosant/1229

DOI : 10.4000/philosant.1229

ISSN : 2648-2789

Éditeur

Éditions Vrin

Édition imprimée

Date de publication : 1 novembre 2011

Pagination : 11-15

ISBN : 978-2-7574-0356-3

ISSN : $1634-4561$

\section{Référence électronique}

Jan Wolenski, « Jan Łukasiewicz, Sur la Logique des stoïciens », Philosophie antique [En ligne], 11 | 2011 , mis en ligne le 01 novembre 2018, consulté le 14 septembre 2020. URL : http:// journals.openedition.org/philosant/1229

\section{(c) (†) $\odot$}

La revue Philosophie antique est mise à disposition selon les termes de la Licence Creative Commons Attribution - Pas d'Utilisation Commerciale - Pas de Modification 4.0 International. 


\section{JAN ŁUKASIEWICZ SUR LA LOGIQUE DES STOÏCIENS Jan WOLENSKI \\ Université Jagellonne, Cracovie}

Jan Łukasiewicz a élaboré un programme de recherche portant sur l'histoire de la logique ${ }^{1}$. À son avis, la logique mathématique contemporaine (modern) est en continuité directe avec la logique formelle du passé. En conséquence, une interprétation correcte et fidèle des idées anciennes exige une application des outils logiques contemporains. Autrement dit, de bonnes études historiques sur la logique doivent consister à examiner la logique d'autrefois à travers les lunettes logiques contemporaines. Łukasiewicz a en particulier accusé des historiens de la logique et de la philosophie célèbres au XIX ${ }^{\mathrm{e}}$ siècle, comme Carl Prantl ou Eduard Zeller, d'avoir mal compris les théories logiques anciennes et médiévales. Certes, il tenait Prantl en grande estime pour son ouvrage monumental intitulé Geschichte der Logik in Abendland, publié dans les années 1855-1870, parce qu'il rassemblait de nombreuses sources capitales. Cependant, Łukasiewicz soutenait que la façon dont Prantl décrit l'histoire de la logique est limitée par des préjugés philosophiques de toutes sortes, propres à la philosophie post-cartésienne. Pour Łukasiewicz, le tournant épistémologique dans la philosophie moderne a corrompu les théories logiques anciennes et médiévales en les infestant d'idées philosophiques superflues et obscures; Leibniz a été le seul à y résister. Ce processus a commencé par Descartes et a culminé avec Kant et Hegel (Prantl lui-même était hégélien), en faisant émerger ce qu’on qualifie de logique philosophique, une mixture de mauvaise philosophie et de logique déformée. L'incapacité de Prantl, tout comme celle des autres historiens de la logique, de voir la différence fondamentale entre la logique aristotélicienne en tant que logique des termes (ou des noms) et celle des stoïciens en tant que logique des propositions est devenue pour Łukasiewicz un exemple typique d'études historiques corrompues par la philosophie. Son projet était simple : il faut récrire l'histoire de la logique à l'aide 
de la logique mathématique, qui devrait alors devenir l'outil méthodologique principal au sein d'une recherche historique sérieuse portant sur les idées logiques anciennes et sur leur développement. « L'histoire de la logique, a-t-il dit, doit être écrite à nouveau, et cela par un historien ayant une maîtrise complète de la logique mathématique ${ }^{2}$.»

Łukasiewicz commença à s'intéresser à la logique stoïcienne au début des années $1920^{3}$. Il a fait une présentation des positions stoïciennes lors du $1^{\text {er }}$ Congrès Philosophique Polonais à Lvov en 1923. Dans un bref sommaire publié quatre ans plus tard, il a exprimé les idées suivantes ${ }^{4}$. Les analyses antérieures de la logique stoïcienne, comme celles proposées par Prantl, n'ont aucune valeur. La logique stoïcienne doit être considérée comme entièrement différente du système aristotélicien : la seconde est une logique des termes alors que la première s'avère être une logique propositionnelle; en conséquence, la logique stoïcienne est plus fondamentale que celle d'Aristote. Enfin, selon Łukasiewicz, les stö̈ciens ont été conscients de cet ordre des systèmes logiques, et, suggérait-il, on pourrait en dire autant de Boèce. En ce qui concerne les sources, Łukasiewicz pensait que le matériel utilisé jusqu'alors était insuffisant ${ }^{5}$. Il s'est penché sur d'autres sources, en particulier sur les écrits de Sextus Empiricus et de Galien, qui, en complétant notre information, éclairent la logique stoïcienne et sa relation avec celle d'Aristote. Łukasiewicz a développé ces idées dans son article célèbre sur l'histoire de la logique des propositions, élaboré à partir de son exposé lors du Praguer Vorkonferenz der Internationalen Kongresse für Einheit der Wissenschaft en $1934^{6}$.

Telle que reconstruite par Łukasiewicz, la logique stoïcienne consiste en des schémas d'inférence ou, en d'autres termes, en des règles d'inférence. C'est un système formalisé bivalent, fondé sur quatre constantes proposi-

2. Łukasiewicz 1934 ; trad. angl. dans Łukasiewicz 1970, p. 198 ; trad. fr. dans Largeault 1972, p. 11.

3. Selon la tradition orale circulant en Pologne, Łukasiewicz a découvert des faits intéressants à propos de la logique de Chrysippe quand, en tant que directeur de thèse, il vérifiait les traductions du grec et du latin dans la thèse de Maria Niedźwiedzka (mieux connue comme Maria Ossowska) consacrée à l'axiologie stö̈cienne, soutenue en 1921.

4. Łukasiewicz 1927.

5. Cela concerne la collection célèbre Stoicorum veterum fragmenta éditée par H. von Arnim en 1903.

6. Łukasiewicz 1934. Entretemps, c'est-à-dire entre 1923 et 1934, Łukasiewicz fit deux remarques sur la logique stoïcienne dans ses textes; $c f$. Łukasiewicz 1929, p. 21-26 = trad. angl. p. 12-14, et Łukasiewicz 1932-1936 = Łukasiewicz 1998, p. 369-370 (le résumé de l'exposé donné à Lvov en 1932). 
tionnelles : négation, implication, conjonction et disjonction, comprises comme fonctions de vérité (ce terme vient de la logique contemporaine [modern]). Dans ce contexte, Łukasiewicz fait allusion à la controverse entre Philon de Mégare et Diodore Cronos (lui aussi un mégarique) concernant la nature de l'implication, où le premier a adopté l'implication matérielle, tandis que le second en a proposé une lecture modale (comme dans le cas de l'implication stricte). Et puisque la logique stoïcienne était vérifonctionnelle, elle optait de son côté pour l'implication matérielle.

Selon Łukasiewicz, les stoïciens ont accepté le schéma suivant comme étant indémontrable (comme étant donc axiomatiquement assumé) :

(1) Si $p$, alors $q$, mais $p$, donc $q$;

(2) Si $p$, alors $q$, mais non- $q$, donc non- $p$;

(3) Non à la fois $p$ et $q$, mais $p$, donc non- $q$;

(4) Soit $p$ ou $q$, mais $p$, donc non- $q$;

(5) Soit $p$ ou $q$, mais non- $q$, donc $p$.

Ces formulations s'écartent des formulations originales, parce que, chez les stoïciens, les variables n'étaient pas des lettres propositionnelles mais des nombres ordinaux; ainsi, (1) se présente dans la version originale comme : «Si le premier, alors le second, mais le premier, donc le second ». Le schéma (1) - (5) a des équivalents évidents dans la logique propositionnelle contemporaine; en particulier, (1) exprime la règle du modus ponens, alors que (2) exprime celle du modus tollens. Par leur réduction aux indémontrables, les stoïciens ont établi un nombre de schémas propositionnels dérivés difficile à estimer de façon précise mais réellement important, selon Łukasiewicz. Il qualifie les exemples connus de ce type de réduction de « chefs-d'œuvre de perspicacité logique ». L'image du développement ultérieur de la logique proposée par Eukasiewicz est la suivante. Continuée au Moyen Âge comme une théorie des conséquences, la logique stoïcienne a été ensuite négligée pour les raisons citées plus haut. C'est Frege qui est revenu (bien qu'inconsciemment) sur le chemin logique inauguré par les stoïciens, consistant à construire le calcul propositionnel comme un système axiomatique. La logique stoïcienne telle qu'elle a été reconstruite par Łukasiewicz est pour lui la preuve principale que la logique formelle au sens contemporain est apparue déjà dans l'Antiquité. 
L'analyse de la logique stoïcienne proposée par Łukasiewicz est devenue très populaire parmi les historiens de la logique ${ }^{7}$. Ses conclusions principales ont été reprises et confirmées par Benson Mates dans son étude exhaustive de la logique stoïcienne, en s'appuyant sur des données textuelles supplémentaires ${ }^{8}$. Lui aussi voyait cette logique comme un premier système de calcul propositionnel. Contrairement à Łukasiewicz qui ne s'intéressait pas à la sémantique des stoïciens, Mates montre les connexions entre leur théorie des signes et leur logique, ce qui fait que la manière dont il saisit cette logique est davantage enracinée dans la philosophie stoïcienne du langage que l'analyse de Łukasiewicz. La différence principale entre Mates et Łukasiewicz concerne les indémontrables. Les deux auteurs s'accordent pour dire que les stoïciens distinguaient cinq indémontrables, mais ils diffèrent quant à la nature de ces schémas. En effet, pour Łukasiewicz les indémontrables devaient être considérés comme des axiomes, alors que pour Mates il s'agit plutôt d'items qui ne nécessitent pas de preuves, puisqu'il est évident que ce sont des principes corrects. Cependant, la direction prise par Łukasiewicz et par Mates a fait l'objet de critiques. L'une a été notamment exprimée par Charles Kahn et reprise ensuite par O'Toole et Jennings'. Kahn soutient en effet que la logique stoïcienne ne peut pas être comprise correctement si on l'interprète comme une anticipation du calcul propositionnel contemporain. Selon lui, une image plus adéquate des théories logiques avancées par les stoïciens nécessite un contexte plus large, où leur épistémologie, leur philosophie du langage, leur éthique et leur cosmologie même soient présentes. O'Toole et Jennings ajoutent des observations plus spécifiques, en remarquant notamment que seule une partie des textes disponibles justifie l'idée selon laquelle les stoïciens comprenaient les connecteurs comme des constantes logiques au sens contemporain. Quoi qu'il en soit, la reconstruction de la logique stoïcienne offerte par O’Toole et Jennings considère les questions formelles comme secondaires et se concentre avant tout sur les aspects informels ${ }^{10}$.

Traduit de l'anglais par Anna Zielinska

7. L'article de Eukasiewicz sur l'histoire de la logique des propositions représentait pour Scholz les vingt plus belles pages écrites sur l'histoire de la logique ; $c f$. Łukasiewicz, Journal (non publié; l'original de ce document se trouve dans les archives de l'Université de Varsovie), p. 25.

8. Mates 1953.

9. Kahn 1969.

10. Il n'est pas dans mon propos d'arbitrer cette controverse relative à la manière dont il faut pratiquer l'histoire de la logique. Je déclare cependant pencher du côté de Łukasiewicz. 


\section{BIBLIOGRAPHIE}

CORCORAN, J. 1974: « Aristotle's natural deduction system », dans J. Corcoran (éd.), Ancient Logic and its Modern Interpretations, Dordrecht, 1974, p. 85-131.

FREDE, M. 1974 : Die stoische Logik, Göttingen, 1974.

KaHN, Ch. 1969 : «Stoic logic and Stoic logos », Archiv für Geschichte der Philosophie, 51 (1969), p. 158-172.

Largeault, J. (éd.) 1972: Logique Mathématique. Textes, Paris, 1972 (Collection U. Épistémologie).

ŁUKASIEWICZ, J. 1927 : «O logice stoików » (« Sur la logique des stoïciens »), Przeglad Filozoficzny, 30 (1927), p. 278-279. [Réimpr. dans Łukasiewicz 1998, p. 368-369 et, avec trad. fr. par A.Zielinska, «Sur la logique des stoïciens », Philosophie antique, 11 (2011), p. 8-9.]

- 1929: Elementy logiki matematycznej («Éléments de logique mathématique »), Varsovie, 1929 (Komisja Wydawnicza Koła Matematyczno-Fizycznego Słuchaczów Uniwersytetu Warszawskiego). [Trad. angl. par O. Wojtasiewicz, Oxford, 1963.]

- 1932-1936: «Z dziejów logiki starożytnej 》 (《Sur l'histoire de la logique ancienne »), Ruch Filozoficzny, 13 (1932-1936), p. 46-47 (= Eukasiewicz 1998, p. 369-370).

- 1934: «Z historji logiki zdań », Przeglad Filozoficzny, 37 (1934), p. 417-437. [Trad. allemande de l'auteur, «Zur Geschichte der Aussagenlogik», Erkenntnis, 5 (1935), p. 111-131 ; trad. angl. dans Łukasiewicz 1970, p. 197-217; trad. fr. par J. Largeault, «Contribution à l'histoire de la logique des propositions » dans Largeault 1972, p. 10-25.]

- 1950 : Aristotle's Syllogistic from the Standpoint of Modern Formal Logic, Oxford, 1950, $2^{\circ}$ édition posthume augmentée, 1956 ; trad. fr. par F. Zaslawsky, La Syllogistique d'Aristote dans la perspective de la logique formelle moderne, Paris, 1972, 2 éd. Paris, 2010.

- 1970 : Selected Works, ed. by L. Borkowski, Amsterdam-Londres-Varsovie, 1970 (Studies in logic and the foundations of mathematics).

- 1998: Logika i metafizyka («Logique et métaphysique »), Varsovie, 1998.

MATES, B. 1953 : Stoic Logic, Berkeley, 1953.

WOLENSKI, J. 1989 : Logic and Philosophy in the Lvov-Warsaw School, Dordrecht, 1989. 